\title{
The TAFs of TFIID Bind and Rearrange the Topology of the TATA-Less RPS5 Promoter
}

\author{
Sarah N. Le ${ }^{1,2}$, Christopher R. Brown ${ }^{3,4}$, Stacy Harvey ${ }^{3,5}$, Hinrich Boeger ${ }^{3}$, Hans Elmlund ${ }^{1,2, *}$ \\ and Dominika Elmlund 1,2,*(D) \\ 1 Department of Biochemistry and Molecular Biology, Biomedicine Discovery Institute, Monash University, \\ Clayton, VIC 3800, Australia \\ 2 ARC Centre of Excellence for Advanced Molecular Imaging, Clayton, VIC 3800, Australia \\ 3 Department of Molecular, Cell and Developmental Biology, University of California, \\ Santa Cruz, CA 95064, USA \\ 4 Alnylam Pharmaceuticals, 300 Third St. Cambridge, MA 02142, USA \\ 5 Two Pore Guys, 2161 Delaware Ave. Suite B, Santa Cruz, CA 95060, USA \\ * Correspondence: hans.elmlund@monash.edu (H.E.); dominika.elmlund@monash.edu (D.E.); \\ Tel.: +61-(0)426-271-213 (H.E.); +61-(0)450-899-171 (D.E.)
}

Received: 9 April 2019; Accepted: 2 July 2019; Published: 4 July 2019

check for updates

\begin{abstract}
The general transcription factor TFIID is a core promoter selectivity factor that recognizes DNA sequence elements and nucleates the assembly of a pre-initiation complex (PIC). The mechanism by which TFIID recognizes the promoter is poorly understood. The TATA-box binding protein (TBP) is a subunit of the multi-protein TFIID complex believed to be key in this process. We reconstituted transcription from highly purified components on a ribosomal protein gene (RPS5) and discovered that TFIID $\triangle$ TBP binds and rearranges the promoter DNA topology independent of TBP. TFIID $\triangle T B P$ binds $\sim 200 \mathrm{bp}$ of the promoter and changes the DNA topology to a larger extent than the nucleosome core particle. We show that TBP inhibits the DNA binding activities of TFIID $\triangle$ TBP and conclude that the complete TFIID complex may represent an auto-inhibited state. Furthermore, we show that the DNA binding activities of TFIID $\triangle T B P$ are required for assembly of a PIC poised to select the correct transcription start site (TSS).
\end{abstract}

Keywords: TBP-associated factor; TAF complex; DNA topology; housekeeping gene transcription; ribosomal protein 5

\section{Introduction}

About sixty proteins, including RNA polymerase II (Pol II) and the general transcription factors (GTFs), assemble at each promoter prior to each round of transcription [1,2]. This so-called pre-initiation complex (PIC) recognizes the promoter, selects a transcription start site (TSS), and synthesizes a nascent transcript. PIC formation is the key step for regulation of gene expression and represents the end point of many signaling pathways.

Pol II promoters either contain or lack a TATA-box consensus [3]. Genes with the TATA-box promoter are often under stringent regulation to enable a rapid response to cellular stress. However, most ( 80\%) Pol II promoters do not contain a canonical TATA-box [3-6]. These TATA-less genes are constitutively transcribed and frequently involved in "housekeeping" processes, essential for all basic cellular maintenance.

The crystal structure of the TATA-box binding protein (TBP) bound to the adenovirus major late TATA promoter revealed a saddle-shaped TBP molecule, stabilizing an $\sim 80^{\circ}$ kink of the promoter by binding the partially unwound TATA-box DNA helix [7]. That structure established a view of the 
TBP-promoter complex as a nucleation point for PIC assembly. However, transcription from Pol II promoters rarely involves the classical TATA-box. TBP is nevertheless essential for transcription of all genes. Analysis of in vivo expression levels in yeast strains with mutations in the DNA binding surface of TBP addressed the question of whether the DNA binding activity of TBP is required for transcription from TATA-less promoters [8]. Most mutants did not support transcription from TATA-containing promoters, whereas TATA-less promoters were largely unaffected. The specific DNA binding activity of TBP appears not to be important for the transcription of "housekeeping" genes with TATA-less promoters. Other components of the general transcription machinery must therefore provide the nucleation point for transcription initiation on TATA-less promoter types.

The general transcription factor TFIID is a mega-Dalton sized complex composed of TBP and 14 TBP-associated factors (TAFs). Previous in vivo studies position TFIID exclusively on TATA-less promoters $[6,9,10]$. However, recent findings position TFIID on both classes of promoters [11,12]. TFIID binds $\sim 200$ base pairs (bp) of DNA upstream and downstream of a cluster of 5-7 TSSs in vivo $[10,13]$. Despite many decades of TFIID research, there are no DNase I footprints that agree with the extended DNA binding observed in vivo. Published transcription assays are generally supplemented with whole cell extracts or utilize non-native TATA-box promoters [14-19].

TBP dynamically associates with the complex of TAFs (TFIID $\triangle$ TBP) and as a result purified TFIID has $\sim 50 \%$ TBP occupancy $[17,20]$. No specific functional roles have been attributed to either TAF complexes containing or lacking TBP in vitro. However, it has been reported that recruitment of TAFs to TATA-less promoters does not require TBP in vivo, raising the possibility that TAFs might be constitutively bound to the core promoter during active transcription [6,21].

In this study, we tested the hypothesis that the TAF complex (TFID $\triangle T B P$ ) recognizes the TATA-less promoter of the ribosomal protein 5 gene (RPS5) independent of TBP in vitro. We found that the TAF complex binds $\sim 200$ bp of the promoter and dramatically changes the DNA topology. Unexpectedly, we also found that TBP inhibits the DNA binding activities of the TAF complex. Our data suggest that binding of TBP to the TAFs of TFIID represents a regulatory switch. TFIID may represent an auto-inhibited state, incapable of binding the promoter and rearranging its topology. Instead, it is the TBP-lacking TAF complex that binds the promoter and rearranges its topology, thereby providing the nucleation point for assembly of a PIC poised to select the correct TSS. We propose that the TAFs-DNA rather than the TBP-DNA complex represents the nucleation point for PIC assembly on the TATA-less RPS5 promoter.

\section{Results}

\subsection{Purification of TFIID $\triangle T B P$ (TAF Complex)}

A TAF complex fully depleted of TBP has not previously been purified to homogeneity. We surmounted this challenge and developed a purification protocol that yielded the complete Saccharomyces cerevisiae TAF complex, containing TAF1-TAF14 in stoichiometric amounts (Figure 1a and Supplementary Figure S1) with less than 3\% TBP contamination, as measured by immunoblotting (Figure 1b). The presence of all 14 TAFs was confirmed by mass spectrometry (Supplementary Figure S2). Electrophoretic mobility gel-shift assay (EMSA) revealed that the TAF complex binds the RPS5 promoter with $\mathrm{nM}$ affinity (Figure 2a).

We showed that TBP alone binds RPS5, forming at least two specific complexes with one or more TBP molecules bound, which agrees with previously published DNAse I footprinting of TBP on the RPS5 promoter [8] (Supplementary Figure S3). Pre-incubation of a constant amount of TBP with an increasing amount of TAFs before addition of DNA in our EMSA shows that TBP has an inhibitory effect on the DNA binding activity of the TAFs. A slight shift was observed with TAFs and TBP present in equimolar amounts (i.e., the complete TFIID complex) (Figure 2b). There was no complete shift at $20 \mathrm{nM}$ of TAF in the presence of $40 \mathrm{nM}$ of TBP, but rather a smear of the free DNA label, which we interpret as free TBP binding to the probe (see Supplementary Figure S3). Our EMSA results showed 
that TBP inhibits TAFs-DNA interactions. Only in the presence of saturating amounts of TAFs vs TBP was a clear shift observed (Figure $2 b$ ).

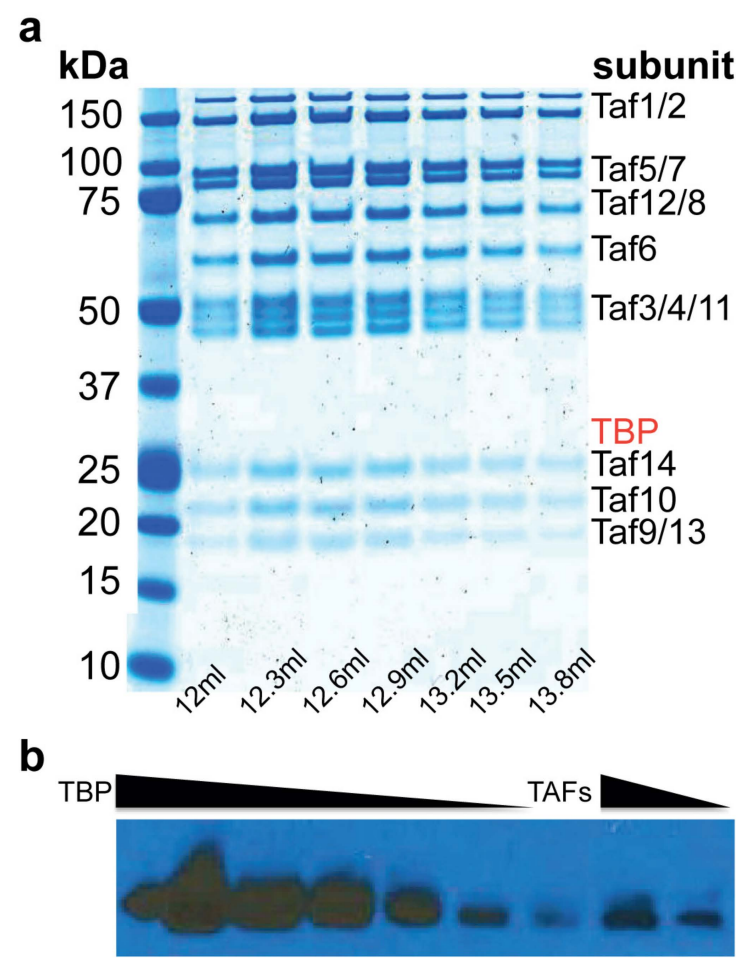

Figure 1. Purification of the TATA-box binding protein (TBP)-associated factor (TAF) complex. (a) SDS-PAGE of TAF complex fractions from size-exclusion chromatography. Elution volumes indicated. (b) Quantification of TBP in the TAF complex preparation by immunoblotting against known amounts of TBP revealed less than 3\% TBP contamination (a two-fold dilution series of recombinant TBP from 2 pmoles to 12.5 fmols and 2 and 1 pmols of purified TAF complex were loaded on the gel).

\subsection{The TAFs Alone Bind and Introduce Significant Topological Changes to the TATA-Less RPS5 Promoter}

To further characterize the TAFs-DNA interactions, we measured the coverage of DNA by the TAF complex using a DNase I footprinting assay with $240 \mathrm{bp}$ of RPS5 (160 bp upstream and $80 \mathrm{bp}$ downstream of the TSS). We observed an extended footprint, spanning almost the entire length of the promoter (Figure 2c). Binding of the TAFs to DNA both upstream and downstream of the TSS was consistent with previous in vivo studies $[10,13]$. We concluded that the TAF complex alone binds a large part ( $200 \mathrm{bp}$ ) of the RPS5 promoter independent of TBP.

Finally, we integrated RPS5 into a plasmid and measured the DNA topological changes introduced by the TAF complex, TBP, and TFIID, respectively, using a topoisomerase I assay (Figure 2d,e; the centers of the distributions are indicated with arrows). Protein binding to a supercoiled plasmid affects DNA topology by changing the local DNA twist, which destabilizes the duplex and generates denatured regions of DNA. In our experiments, analysis of the topoisomer distribution for the TAF complex revealed a large linking number change $(\Delta \mathrm{Lk})$ of +1.91 . Thus, the TAFs alone change the promoter DNA topology to a larger extent than the nucleosome core particle, which introduces a $\Delta$ Lk of -1 to -1.25 [22]. The opposite sign of the $\Delta$ Lk suggests that the TAF complex wraps DNA in the opposite direction to the nucleosome or increases the twist of the DNA. TBP introduced a small negative $\Delta \mathrm{Lk}$, consistent with previous reports [6], and local untwisting of DNA by TBP. Incubating the plasmid with TFIID (TAFs and TBP in equimolar amounts mixed before the addition of plasmid DNA) reduced $\Delta \mathrm{Lk}$ to +0.48 . The similarity between TBP and TFIID topoisomer distribution is consistent with our other results, which show that TBP interferes with TAFs binding to the promoter. In the topoisomerase experiment, we added saturating amounts of proteins vs plasmid. In the TFIID sample there are 
three different species (TBP, TAFs, and TAFs-TBP), which compete for the promoter DNA. The wider distribution in the TFIID sample can therefore be explained by greater conformational and structural heterogeneity. The much wider topoisomer distribution of the TAFs sample vs TBP and TFIID shows that TAFs alone can bind and rearrange the RPS5 promoter topology without TBP, and addition of TBP affects this behavior. The strong band at the top of the gel corresponds to the nicked plasmid.
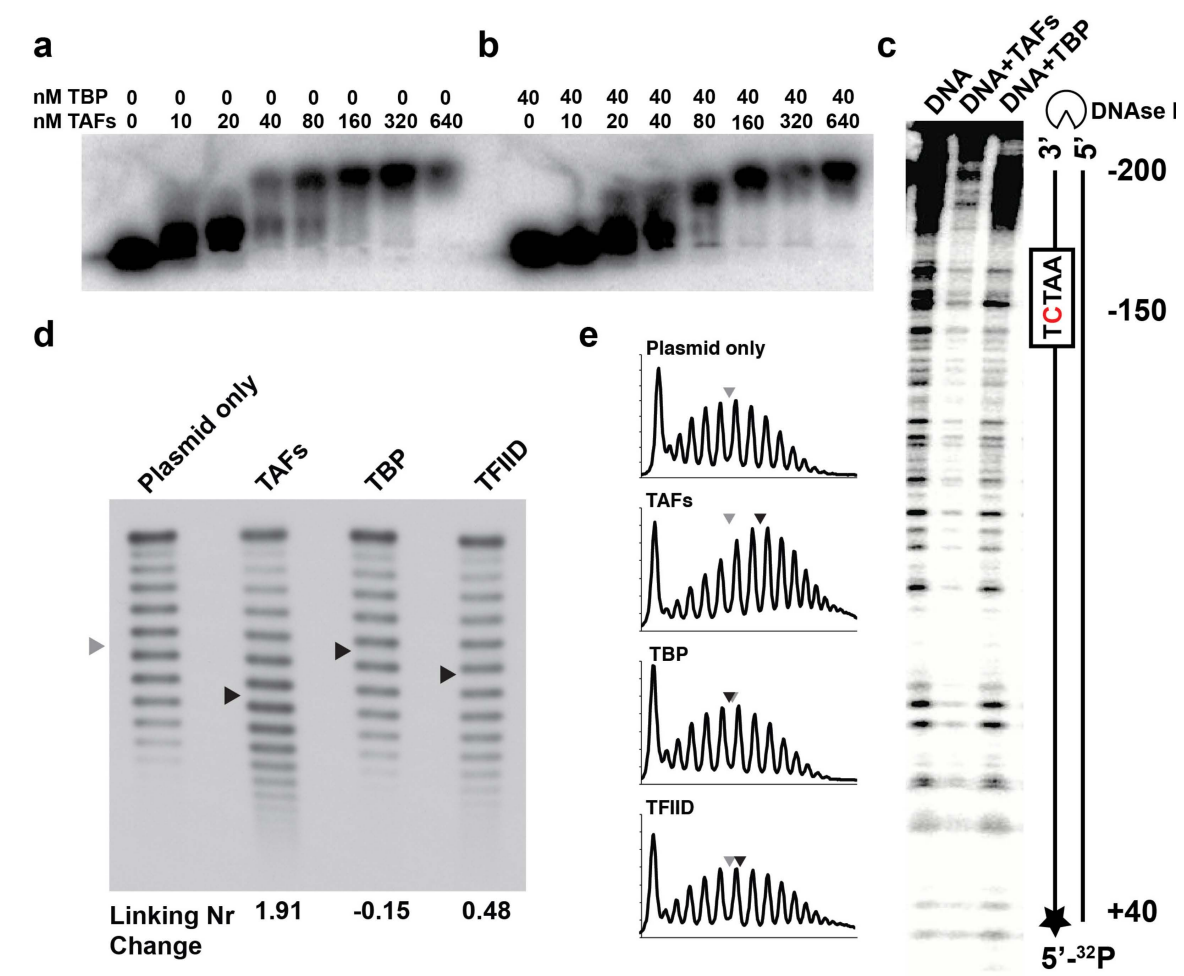

Figure 2. DNA binding experiments. (a) Electrophoretic mobility gel-shift assay (EMSA) in $0.8 \%$ Mg-agarose gel showed that the TAF complex binds the RPS5 promoter with nM affinity. (b) EMSA with addition of a constant amount of TBP while titrating TAFs. (c) DNAse I footprinting assay with 240 bp of RPS5 promoter. The TAF complex introduced an extended footprint, spanning 200 bp DNA. TBP introduced a small footprint in the region around the pseudo-TATA-box. (d) Topoisomerase I assay showed a fourfold increase in positive supercoiling for TAFs vs TFIID. The centers of the distributions are indicated with arrows. (e) Intensity distribution profiles of the bands in (d).

\subsection{The TAF Complex Provides the Nucleation Point for Correct Housekeeping Gene Transcription In Vitro}

In vitro run-off transcription from the HIS4 TATA promoter produces mRNAs of length consistent with a TSS located 25 bp downstream of the TATA-box [19,20] (Supplementary Figure S4). On TATA-less promoters, the distance between the pseudo-TATA-box and the cluster of 5-7 in vivo TSSs [13] is typically much longer and more variable ( $\sim 0$ bp on RPS5; Supplementary Figure S5).

We reconstituted the housekeeping transcription reaction from highly purified components (Figure 3c). First, we established that the purified factors (omitting the TAFs) were active by performing the run-off transcription assay on the HIS4 TATA promoter (Supplementary Figure S4; lane 1), as described previously $[23,24]$. Next, we showed that TBP alone, in the absence of TAFs, was capable of nucleating a PIC and initiating transcription from a non-native TSS on RPS5 (Figure 3a; lane 3 and Supplementary Figure S4; lane 3). In both assays, a strong band was observed corresponding to a non-native transcript of around $140 \mathrm{bp}$, which was located approximately $25 \mathrm{bp}$ downstream from the pseudo-TATA box. The characteristic cluster of specific RPS5 mRNA products [9] was detected only with TAFs present in the reaction (Figure 3a; lanes 2,3). The presence of a strong TBP-dependent artificial mRNA product in all reactions suggests that a population of TAF-containing and TAF-lacking 
PICs was formed in vitro. The TAF-lacking PIC was presumably formed through an assembly pathway similar to that characterized for the HIS4 TATA promoter [23,24].

a

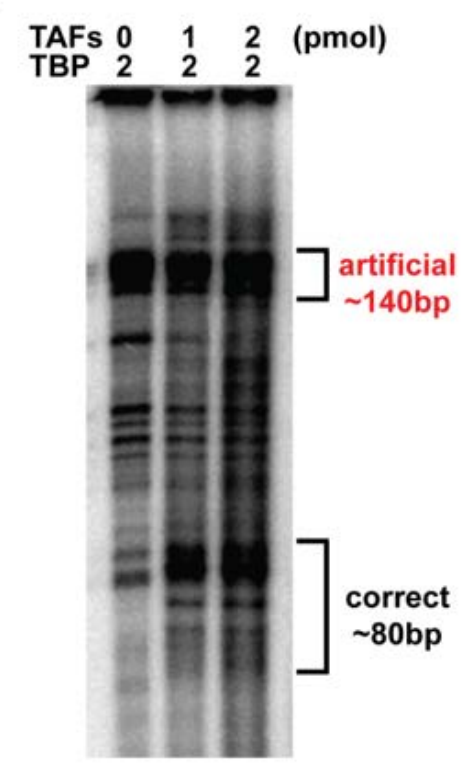

b

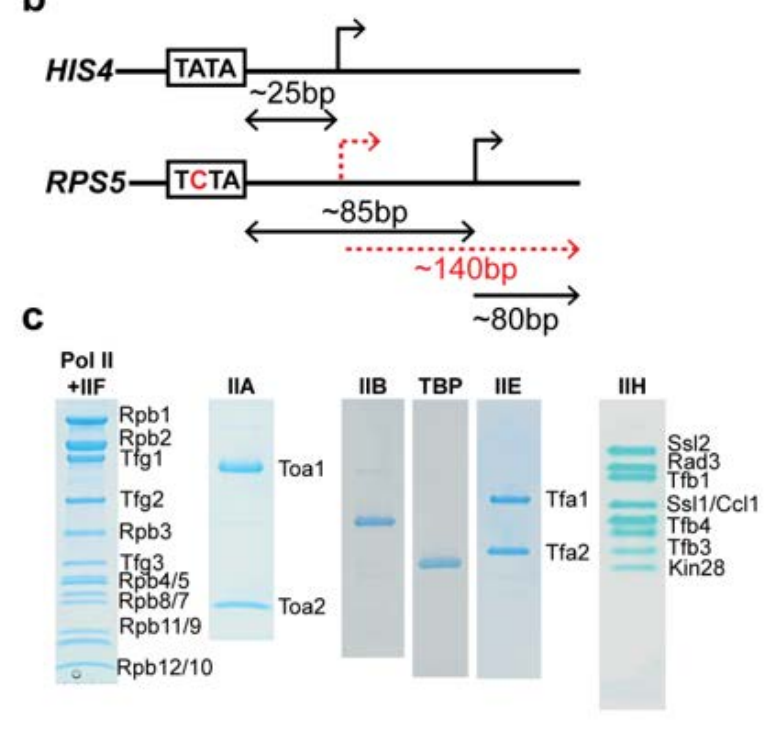

Figure 3. Reconstitution of transcription from the housekeeping RPS5 gene. (a) Run-off transcription gel. (b) Schematic representation of the TATA-containing HIS4 and the TATA-less RPS5 promoters. The artificial TSS due to TBP-dependent not TAF-dependent transcription is indicated with a dashed red arrow. The length of the artificial and correct transcripts on the RPS5 promoter are indicated with a red dashed and black arrow below the RPS5 promoter schematic picture. (c) Purification of general transcription factors (GTFs). SDS-PAGE of the remaining purified GTFs with the individual subunits indicated for multi-subunit GTFs.

\subsection{Structural Rearrangements of DNA Bound and Unbound TAF Complexes}

We sought to solve the cryo-electron microscopy (EM) structures of apo-TAFs and TAFs-DNA, to understand at the molecular level where the TAFs contact promoter DNA and what topological changes are introduced. Three-dimensional reconstruction of both apo-TAFs and TAFs-DNA complexes stalled at $\sim 20 \AA$. We concluded that further stabilization of both complexes was required as the inherent heterogeneity present in both samples posed a real challenge for further high-resolution studies. Preliminary 3D reconstructions indicated visually distinct differences between the DNA bound and unbound TAF complexes. The apo-TAF complex showed the expected horseshoe-shaped 3-lobed structure, consistent with previous studies (Figure 4a) [25]. In contrast, when the TAFs were bound to $\sim 200 \mathrm{bp}$ of promoter DNA, the molecule appeared to adopt a more globular nucleosome-like structure, with the DNA path wrapping the entire molecule, presumably to bring the TSS closer to the pseudo-TATA-box (Figure 4b).

We did not observe protruding DNA ends, neither in class averages nor in the $3 \mathrm{D}$ reconstructions. The apo structure was $220 \AA$ in the longest direction and the DNA bound structure was compacted to $180 \AA$. The DNA used in the EM studies was $\sim 200 \mathrm{bp}$, which corresponded to $680 \AA$ A. The extended footprint of around $200 \mathrm{bp}$ (Figure 2c) suggested that the DNA was wrapped around the TAFs. Furthermore, the large linking number change $(\Delta \mathrm{Lk})$ of +1.91 introduced by the TAFs, which was almost double that of the nucleosome, suggested extensive DNA wrapping. Visualization of the mobility of the different lobes and how this mobility was coordinated to orchestrate the conformational changes required to accomplish transcription initiation by the TAFs of TFIID remains a challenge. 
a

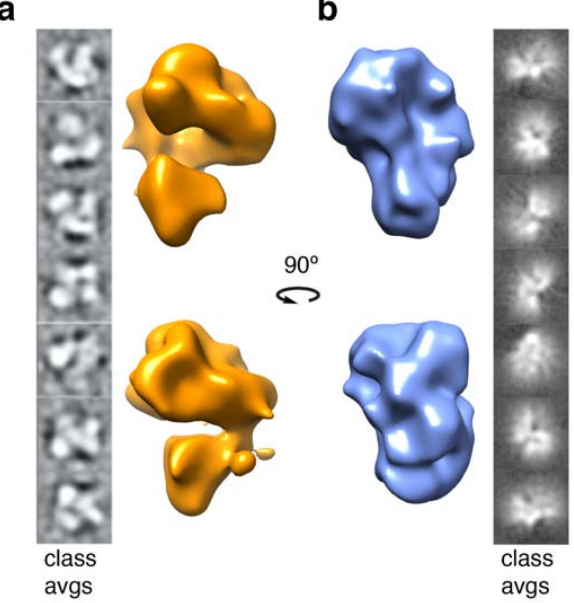

Figure 4. Preliminary 3D reconstructions and corresponding class averages of apo-TAFs and TAFs-DNA complexes. (a) apo-TAF complex (orange) showing the distinct horseshoe-shaped 3-lobed structure. (b) TAFs in complex with 200 bp of RPS5 promoter (blue) showing a globular nucleosome-like core structure with extra density around the entire molecule, which we attribute to the wrapping of DNA by the TAFs. These preliminarily 3D reconstructions indicate a visually distinct difference between the DNA bound and unbound TAF complexes.

\section{Discussion}

We show that the TAFs of TFIID recognize the RPS5 promoter and rearrange its topology independent of TBP. Our data supports the idea that the TAFs of TFIID act as core promoter selectivity factors independent of TBP in vivo [6]. Unexpectedly, we find that TBP interferes with the DNA binding activity of the TAF complex. When TBP is pre-incubated with TAFs in equimolar amounts (i.e., TFIID) before addition of DNA in the gel shift assay, the bands become smeary and no clear shift is observed. The TAF complex alone, in contrast, produces a clear DNA shift (Figure 2a,b; compare bracketed lanes). Furthermore, the TAF activities responsible for the dramatic change in DNA topology are inhibited in the presence of TBP (Figure 2d; compare lanes "TAFs" and "TFIID").

There are structural data that relate to our findings. Schizosaccharomyces pombe TAFs-DNA, TAF complex, and TFIID cryo-EM reconstructions showed that TBP, when bound to the TAFs, places a molecular lid over the DNA binding furrow of the TAF complex [20]. Furthermore, the NMR structure of the N-terminal domain of TAF1 (TAND) in complex with TBP [26] showed that TAND occupies the very same position as the DNA would in the TBP-DNA crystal structure [7] (Figure 5a,b). The inescapable conclusion is that TBP has two mutually exclusive binding partners within the TAF complex. TBP either interacts with a promoter segment pre-bound to TAFs or, when TAFs are not engaged with DNA, TBP interacts with the TAND domain of TAF1.

Our data suggest that binding of TBP to the TAFs of TFIID represents a regulatory switch. The TFIID molecule represents an auto-inhibited state, incapable of binding and rearranging the promoter DNA topology. A plausible explanation for this phenomenon is that TBP, when bound to the TAFs, inhibits TAFs-DNA interactions [20] (Figure 2b,d,e). Previous studies show that TFIID binds DNA only when TFIIA is present to reverse this inhibition [16]. Furthermore, quantitative analysis of in vivo transcription levels has shown that many activators fused to DNA-binding domains work better in cells with TAND deletions [27]. In light of our findings, that deletion of parts of TAND may decrease the affinity of TBP for TAFs and shift the equilibrium between the TAFs and the auto-inhibited TFIID molecule toward the DNA-binding TAF complex.

All currently available structural data of TFIID in complex with the promoter have been obtained with short DNA sequences. Recent cryo-EM TFIID structures with a promoter segment of $8 \mathrm{bp}$ indicate no wrapping of DNA by the TAFs [25], whereas our preliminary cryo-EM 3D reconstructions show a 
more compact globular structure when TAFs are bound to 200 bp of DNA, presumably due to DNA wrapping (Figure $4 \mathrm{~b}$ ). Taken together, our data show that by using a 240 bp TATA-less RPS5 promoter, the TAFs of TFIID bind and wrap DNA in the opposite direction to the nucleosome core particle.

Our study indicates a role of TAFs beyond simply recognizing promoter DNA. TAFs are required for the assembly of a PIC poised to select the correct TSS on the TATA-less RPS5 promoter. That such an extended footprint and large change to DNA topology is correlated with correct TSS selection is an unexpected finding. Perhaps this substantial change to DNA topology is a hallmark of housekeeping gene transcription.

TBP or other GTFs may bind the TATA-less promoter before TAFs in vivo, followed by recruitment of the TAFs and the remaining GTFs. However, this pathway is unlikely to result in the formation of a PIC poised to select the correct TSS in vitro, since a mixture of TAF-dependent and TBP-independent PICs are formed (Figure 3a). Instead, we conclude that TBP and the other GTFs direct the PIC assembly on RPS5 after the formation of a TAFs-DNA complex (Figure 5c,d). Our findings agree with the recently published human TFIID structure [25], where the authors observe that TAF11 and TAF13 interact with TBP and keep TBP inhibited before the TAFs are bound to the promoter.

We discovered that the TAFs of TFIID introduce a significantly rearranged RPS5 promoter topology, required for correct start-site selection in vitro. Our study lays the foundation for further mechanistic and structural studies of housekeeping transcription, involving co-regulators, activators, and repressors.

a

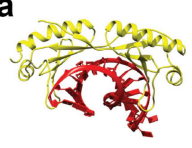

b

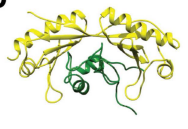

C

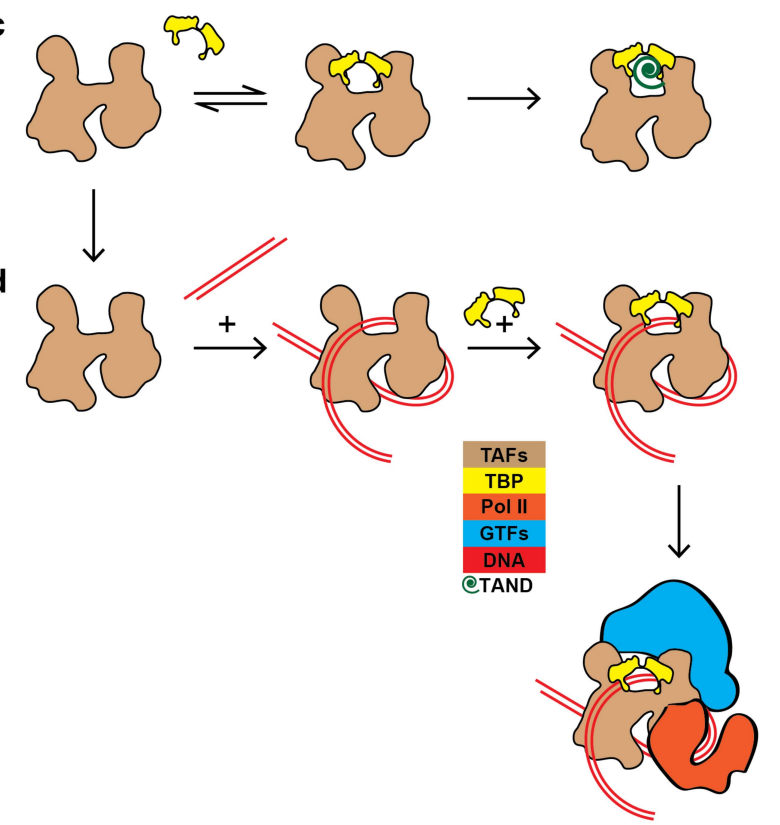

Figure 5. Schematic of TAFs-DNA assembly pathways in vitro. (a) X-ray crystal structure of TBP in complex with TATA-box DNA (PDB:1YTB). (b) NMR structure of TBP in complex with the N-terminal domain of TAF1 (TAND) (PDB:1TBA). (c) Dynamic association of TBP with its TAFs. When TBP is associated with its TAFs, TBP engages with the TAND domain of TAF1 and represents an auto-inhibited TFIID complex unable to bind and recognize promoter DNA. (d) The pre-initiation complex (PIC) assembly pathway that we propose for the TATA-less promoter of the RPS5 gene. When the TAFs are not bound to TBP, or once the TBP-TAFs interaction is broken, DNA binds the TAFs and TBP is then engaged at the promoter on lobe A of TFIID. The TAF complex alone rearranges the DNA topology, providing the nucleation point required for assembly of a PIC poised to select the correct TSS. 


\section{Materials and Methods}

\subsection{Oligonucleotides}

A 240 bp RPS5 (-180/+60) DNA fragment was amplified by PCR from yeast genomic DNA using primers with EcoRV sites. The fragment was subsequently TA-cloned into a $p$ Drive vector. The plasmid construct was amplified in E. coli and purified and digested with EcoRV. The promoter fragment was agarose gel purified, ethanol precipitated, and re-suspended in water. Non-native ATC and GAT were retained at the $5^{\prime}$ - and $3^{\prime}$-ends.

\subsection{Protein Purification}

The TAF complex was purified from a Saccharomyces cerevisiae TAF1-TAP tagged strain. A 36 L yeast culture was grown to OD $=6-7$ and cells harvested by centrifugation. Harvested cells were resuspended in equal volume lysis buffer (100 mM HEPES pH 7.8, $400 \mathrm{mM}$ potassium acetate, $4 \mathrm{mM}$ magnesium sulfate, $10 \%$ glycerol, $10 \mathrm{mM}$ DTT, and protease inhibitor cocktail: PMSF $3.4 \%$ (w/v), leupeptin 5.68\% $(\mathrm{w} / \mathrm{v})$, pepstatin A $0.0274 \%(\mathrm{w} / \mathrm{v})$, and benzamidine $\mathrm{HCl} 6.6 \%(\mathrm{w} / \mathrm{v}))$, and lysed in a DynoMill (Multi Lab WAB, Blaxland, Australia). Cellular debris was removed by centrifugation in a JLA8.1 rotor (Beckman Coulter, Sydney, Australia) at $8000 \times \mathrm{rpm}, 4{ }^{\circ} \mathrm{C}$ for $1 \mathrm{~h}$, and the supernatant was adjusted to $600 \mathrm{mM}$ potassium acetate. To remove nucleic acids, PEI (50\% w/v) was added to the lysate to a final concentration of $0.1 \%$. The lysate was further clarified by ultracentrifugation in a Ti-45 rotor (Beckman Coulter) at $45,000 \times$ rpm for $1 \mathrm{~h}$. The cleared supernatant was loaded onto an IgG column (10 mL of $10 \mathrm{mg} / \mathrm{mL}$ IgG resin). Bound complex was washed with $1 \mathrm{~L}$ of high salt buffer (50 mM HEPES pH 7.8, $1 \mathrm{M}$ potassium acetate, $2 \mathrm{mM}$ magnesium sulfate, 5\% glycerol, $1 \mathrm{mM}$ DTT, and protease inhibitor cocktail), $500 \mathrm{~mL}$ of Low Salt Buffer (50 mM HEPES pH 7.8, $50 \mathrm{mM}$ potassium acetate, $2 \mathrm{mM}$ magnesium sulfate, $5 \%$ glycerol, $1 \mathrm{mM}$ DTT, and protease inhibitors) and equilibrated in $200 \mathrm{~mL}$ TEV protease Buffer (50 mM HEPES pH 7.8, $200 \mathrm{mM}$ potassium acetate, $2 \mathrm{mM}$ magnesium sulfate, $5 \%$ glycerol, and $1 \mathrm{mM}$ DTT). The bound complex was cleaved overnight with $100 \mu \mathrm{g}$ TEV protease at $4{ }^{\circ} \mathrm{C}$ and eluted in 5 column volumes of gel filtration buffer (50 mM HEPES pH 7.8, $400 \mathrm{mM}$ potassium acetate, $4 \mathrm{mM}$ magnesium sulfate, and $1 \mathrm{mM}$ DTT), concentrated and loaded onto a pre-equilibrated Superose $610 / 300 \mathrm{column}$ (GE Healthcare, Melbourne, Australia). The TAF-complex eluted as a single symmetric peak centered at around $12.6 \mathrm{~mL}$. The other GTFs and Pol II were purified as previously described [17,19,20].

\subsection{Quantitation of the Amount of TBP in the TAF Complex Preparation}

Quantitation of the amount of TBP was done by immunoblotting. A two-fold dilution series of recombinant TBP ( 2 pmoles to 12.5 fmols) and 2 and 1 pmols of purified TAF complex from above were loaded on SDS-PAGE and electrophoresed. The proteins were then transferred to a PVDF membrane using a western transfer system (Bio-Rad, Gladesville, Australia). The blot was blocked with 5\% skim milk in TBST, followed by incubation with anti-TBP antibody (Abcam, cat\# ab28175) and secondary antibody (Abcam, San Francisco, CA, USA), washed 3 times with TBST, and developed. The blot was scanned, and the intensities of the bands were quantitated using ImageJ (Version 1.51, NIH).

\subsection{Electrophoretic Mobility Shift Assay}

RPS5 promoter was end-labeled with $\left(\gamma_{-}{ }^{32} \mathrm{P}\right) \mathrm{ATP}$ using T4 polynucleotide kinase. Ten $\mu \mathrm{L}$ binding reactions contained a serial dilution of TAFs, or a serial dilution of TAFs preincubated with a constant amount of TBP, in protein binding buffer (20 mM HEPES, $100 \mathrm{mM}$ potassium acetate, $2 \mathrm{mM}$ magnesium acetate, $2 \mathrm{mM}$ DTT, $10 \mu \mathrm{g}$ of BSA, and 5\% glycerol), and a 10,000 cpm probe (32 fmoles). An unrelated competitor DNA of the same length was added in 100 fold excess to prevent unspecific binding. After $30 \mathrm{~min}$ of incubation at room temperature, reactions were resolved on a $0.8 \%$ agarose gel in 45 $\mathrm{mM}$ boric acid, $5 \mathrm{mM}$ magnesium acetate, and $45 \mathrm{mM}$ Tris. The $4 \mathrm{~mm}$ thick gels were run at $7.5 \mathrm{~V} / \mathrm{cm}$ for $50 \mathrm{~min}$ at $4{ }^{\circ} \mathrm{C}$. Gels were dried, exposed overnight, and quantitation was performed with the use of a PhosphorImager (GE Healthcare, Melbourne, Australia)and ImageQuant software (TL 7.0, 
GE Healthcare, Sweden). The TBP/RPS5 gel shift was performed as described above, and reactions were resolved on a $2 \%$ agarose gel.

\subsection{DNase I Footprinting}

The RPS5 promoter was end-labeled with $\left(\gamma^{-}{ }^{32} \mathrm{P}\right)$ ATP. Five fmol RPS5 was incubated for $20 \mathrm{~min}$ at $30{ }^{\circ} \mathrm{C}$ in protein binding buffer $(25 \mathrm{mM}$ HEPES $\mathrm{pH} 7.8,150 \mathrm{mM}$ potassium acetate, $3 \mathrm{mM}$ magnesium acetate, $7 \%$ glycerol, and $2 \mathrm{mM}$ DTT) alone or with 2 pmol TAFs or TBP. DNase I (0.1 U) was added for $1 \mathrm{~min}$ and reactions were terminated with stop buffer ( $400 \mathrm{mM}$ sodium acetate, $0.2 \% \mathrm{SDS}$, and $10 \mathrm{mM}$ EDTA). Samples were digested with proteinase $\mathrm{K}$, phenol/chloroform extracted, and electrophoresed on an $8 \%$ polyacrylamide/urea gel at $10 \mathrm{~W}$ for $2.5 \mathrm{~h}$. Gels were dried, exposed overnight, and quantitation performed using a PhosphorImager and the ImageQuant software.

\subsection{Topological Analysis}

pDrive/RPS5 was incubated with TAFs, TBP, and TAFs and TBP (TFIID), respectively, in protein binding buffer ( $25 \mathrm{mM}$ HEPES $\mathrm{pH} 7.8,150 \mathrm{mM}$ potassium acetate, $3 \mathrm{mM}$ magnesium acetate, $7 \%$ glycerol, $2 \mathrm{mM}$ DTT) for $20 \mathrm{~min}$ at $30^{\circ} \mathrm{C}$. Topoisomerase I ( $\left.1 \mathrm{U}\right)$ was added and samples were incubated at $37^{\circ} \mathrm{C}$ for $1 \mathrm{~h}$, followed by addition of $0.5 \%$ SDS and digestion with proteinase $\mathrm{K}$. DNA samples were purified with a Zymo DNA clean/concentrator Kit. Analysis of topoisomer distributions was performed as previously described [28], except DNA was electrophoresed in a $0.9 \%$ agarose gel containing $8 \mu \mathrm{M}$ chloroquine at $1.2 \mathrm{~V} / \mathrm{cm}$ for $42 \mathrm{hr}$, blotted, and hybridized with radiolabeled DNA probe (against RPS5) as previously described [24].

\subsection{Run-Off Transcription Assay}

One pmol of RPS5 template was mixed with 0,1 , and 2 pmol of TAFs, in $2 \mu \mathrm{L}$ of transcription buffer (50 mM HEPES $\mathrm{pH}$ 7.6, $300 \mathrm{mM}$ potassium acetate, $5 \mathrm{mM}$ magnesium sulfate, $5 \%$ glycerol, and $5 \mathrm{mM}$ DTT) and incubated for $15 \mathrm{~min}$ on ice, followed by addition of 2 pmol of TBP and further incubation for $15 \mathrm{~min}$. Then, 2 pmols of TFIIA, $-\mathrm{B},-\mathrm{E}$, and $-\mathrm{H}$ were added to the reaction mixture and the potassium acetate concentration was adjusted to $90 \mathrm{mM}$ followed by $15 \mathrm{~min}$ incubation on ice. Finally, 1 pmol Pol II-TFIIF complex was added and potassium acetate concentration was further diluted to $30 \mathrm{mM}$ and magnesium sulfate adjusted to $7.5 \mathrm{mM}$ in $20 \mu \mathrm{L}$ total reaction volume. Transcription was initiated by adding an equal volume of $2 X$ transcription mixture (1.6 mM ATP, $1.6 \mathrm{mM}$ GTP, $1.6 \mathrm{mM}$ CTP, $40 \mu \mathrm{M}$ UTP, $0.083 \mu \mathrm{M}\left(\alpha{ }^{3}{ }^{32} \mathrm{P}\right) \mathrm{UTP}(2.5 \mu \mathrm{Ci}), 10 \mathrm{mM}$ magnesium acetate, and 5 units of RNaseOUT) at $30^{\circ} \mathrm{C}$. The reaction was stopped after $20 \mathrm{~min}$ by addition of $185 \mu \mathrm{L}$ of stop buffer $(10 \mathrm{mM}$ Tris $\mathrm{pH}$ 7.5, $300 \mathrm{mM}$ sodium acetate $\mathrm{pH} 5.5,5 \mathrm{mM}$ EDTA, $0.7 \% \mathrm{SDS}, 0.1 \mathrm{mg} / \mathrm{mL}$ glycogen, and $0.013 \mathrm{mg} / \mathrm{mL}$ proteinase $\mathrm{K})$. mRNA products were ethanol precipitated, resuspended in loading buffer, and resolved on a denaturing $6 \%$ polyacrylamide/urea gel. Gels were exposed overnight and quantitation was performed using a PhosphorImager and the ImageQuant software. Control reactions using HIS4 $(-96 /+112)$ were performed as described above, but without the addition of TAFs.

\subsection{D Reconstructions of Apo-TAFs and TAFs-DNA Complexes}

For cryo-EM sample preparation, purified TAF complex was stabilized using the GraFix method [29,30] and DNA was added in 2x molar excess and incubated for $15 \mathrm{~min}$ at room temperature for the TAFs-DNA complex. Then, $4 \mu \mathrm{L}$ of each protein sample was applied to a Quantifoil R1.2/1.3 holey carbon grid and rapidly frozen in liquid ethane using a Vitrobot IV (FEI). The sample-containing grid was then loaded into the Titan Krios FEG-TEM $300 \mathrm{kV}$ instrument equipped with a K2 direct electron detector (Gatan) and imaged under low-dose conditions (10-15 e/ $\left./ \AA^{2}\right)$. Collected movies were motion and CTF corrected using SIMPLE [31,32], and particles were manually picked using EMAN2 [33]. Data processing was performed using SIMPLE [31,32], and depictions of 3D reconstructed models were generated using UCSF Chimera [34]. 
Supplementary Materials: Supplementary materials can be found at http://www.mdpi.com/1422-0067/20/13/ 3290/s1.

Author Contributions: Conceptualization: D.E. and H.E.; supervision: D.E., H.E., and H.B.; methodology and investigation: S.N.L., C.R.B., and S.H.; formal analysis: S.N.L., C.R.B., S.H., H.B., H.E., and D.E.; writing一original draft preparation: S.N.L.; writing - review and editing: D.E., H.E., and H.B.; software: H.E. and D.E.; visualization: S.N.L. and C.B.; project administration: D.E. and H.E.; funding acquisition: H.E.

Funding: This research was funded by the Australian Research Council (ARC), grant number DP170101850.

Conflicts of Interest: The authors declare no conflict of interest.

\begin{tabular}{ll}
\multicolumn{2}{l}{ Abbreviations } \\
bp & Base pairs \\
EMSA & Electrophoretic mobility gel-shift assay \\
GTFs & General transcription factors \\
$\Delta$ Lk & Linking number change \\
PIC & Pre-initiation complex \\
Pol II & RNA polymerase II \\
RPS5 & Ribosomal protein 5 \\
TAFs & TBP-associated factors \\
TAND & N-terminal domain of TAF1 \\
TBP & TATA-box binding protein \\
TSS & Transcription start site
\end{tabular}

\section{References}

1. Conaway, R.C.; Conaway, J.W. General initiation factors for RNA polymerase II. Annu. Rev. Biochem. 1993, 62, 161-190. [CrossRef] [PubMed]

2. Kornberg, R.D. The molecular basis of eukaryotic transcription. Proc. Natl. Acad. Sci. USA 2007, 104, 12955-12961. [CrossRef] [PubMed]

3. Basehoar, A.D.; Zanton, S.J.; Pugh, B.F. Identification and distinct regulation of yeast TATA box-containing genes. Cell 2004, 116, 699-709. [CrossRef]

4. Yang, C.; Bolotin, E.; Jiang, T.; Sladek, F.M.; Martinez, E. Prevalence of the initiator over the TATA box in human and yeast genes and identification of DNA motifs enriched in human TATA-less core promoters. Gene 2007, 389, 52-65. [CrossRef]

5. Lee, D.H.; Gershenzon, N.; Gupta, M.; Ioshikhes, I.P.; Reinberg, D.; Lewis, B.A. Functional characterization of core promoter elements: The downstream core element is recognized by TAF1. Mol. Cell. Biol. 2005, 25, 9674-9686. [CrossRef] [PubMed]

6. Li, X.Y.; Bhaumik, S.R.; Green, M.R. Distinct classes of yeast promoters revealed by differential TAF recruitment. Science 2000, 288, 1242-1244. [CrossRef]

7. Kim, Y.; Geiger, J.H.; Hahn, S.; Sigler, P.B. Crystal structure of a yeast TBP/TATA-box complex. Nature 1993, 365, 512-520. [CrossRef]

8. Kamenova, I.; Warfield, L.; Hahn, S. Mutations on the DNA binding surface of TBP discriminate between yeast TATA and TATA-less gene transcription. Mol. Cell. Biol. 2014, 34, 2929-2943. [CrossRef]

9. Kuras, L.; Kosa, P.; Mencia, M.; Struhl, K. TAF-Containing and TAF-independent forms of transcriptionally active TBP in vivo. Science 2000, 288, 1244-1248. [CrossRef]

10. Rhee, H.S.; Pugh, B.F. Genome-wide structure and organization of eukaryotic pre-initiation complexes. Nature 2012, 483, 295-301. [CrossRef]

11. Warfield, L.; Ramachandran, S.; Baptista, T.; Devys, D.; Tora, L.; Hahn, S. Transcription of nearly all yeast RNA polymerase II-transcribed genes is dependent of transcription factor TFIID. Mol. Cell 2017, 68, 118-129. [CrossRef]

12. Vinayachandran, V.; Reja, R.; Rossi, M.J.; Park, B.; Rieber, L.; Mittal, C.; Mahony, S.; Pugh, B.F. Widespread and precise reprogramming of yeast protein-genome interactions in response to heat shock. Genome Res. 2018, 28, 357-366. [CrossRef] 
13. Sugihara, F.; Kasahara, K.; Kokubo, T. Highly redundant function of multiple AT-rich sequences as core promoter elements in the TATA-less RPS5 promoter of Saccharomyces cerevisiae. Nucleic Acids Res. 2011, 39, 59-75. [CrossRef]

14. Pugh, B.F.; Tjian, R. Transcription from a TATA-less promoter requires a multisubunit TFIID complex. Genes Dev. 1991, 5, 1935-1945. [CrossRef] [PubMed]

15. Zhou, Q.; Lieberman, P.M.; Boyer, T.G.; Berk, A.J. Holo-TFIID supports transcriptional stimulation by diverse activators and from a TATA-less promoter. Genes Dev. 1992, 6, 1964-1974. [CrossRef] [PubMed]

16. Guermah, M.; Tao, Y.; Roeder, R.G. Positive and negative TAF(II) functions that suggest a dynamic TFIID structure and elicit synergy with TRAPs in activator-induced transcription. Mol. Cell. Biol. 2001, 21, 6882-6894. [CrossRef] [PubMed]

17. Sanders, S.L.; Garbett, K.A.; Weil, P.A. Molecular characterization of Saccharomyces cerevisiae TFIID. Mol. Cell. Biol. 2002, 22, 6000-6013. [CrossRef]

18. Liu, W.L.; Coleman, R.A.; Grob, P.; King, D.S.; Florens, L.; Washburn, M.P.; Geles, K.G.; Yang, J.L.; Ramey, V.; Nogales, E.; et al. Structural changes in TAF4b-TFIID correlate with promoter selectivity. Mol. Cell 2008, 29, 81-91. [CrossRef]

19. Layer, J.H.; Miller, S.G.; Weil, P.A. Direct transactivator-transcription factor IID (TFIID) contacts drive yeast ribosomal protein gene transcription. J. Biol. Chem. 2010, 285, 15489-15499. [CrossRef]

20. Elmlund, H.; Baraznenok, V.; Linder, T.; Szilagyi, Z.; Rofougaran, R.; Hofer, A.; Hebert, H.; Lindahl, M.; Gustafsson, C.M. Cryo-EM reveals promoter DNA binding and conformational flexibility of the general transcription factor TFIID. Structure 2009, 17, 1442-1452. [CrossRef]

21. Yudkovsky, N.; Ranish, J.A.; Hahn, S. A transcription reinitiation intermediate that is stabilized by activator. Nature 2000, 408, 225-229. [CrossRef] [PubMed]

22. Crick, F.H. Linking numbers and nucleosomes. Proc. Natl. Acad. Sci. USA 1976, 73, 2639-2643. [CrossRef] [PubMed]

23. Murakami, K.; Calero, G.; Brown, C.R.; Liu, X.; Davis, R.E.; Boeger, H.; Kornberg, R.D. Formation and fate of a complete 31-protein RNA polymerase II transcription preinitiation complex. J. Biol. Chem. 2013, 288, 6325-6332. [CrossRef] [PubMed]

24. Murakami, K.; Elmlund, H.; Kalisman, N.; Bushnell, D.A.; Adams, C.M.; Azubel, M.; Elmlund, D.; Levi-Kalisman, Y.; Liu, X.; Gibbons, B.J.; et al. Architecture of an RNA polymerase II transcription pre-initiation complex. Science 2013, 342, 1238724. [CrossRef] [PubMed]

25. Patel, A.B.; Louder, R.K.; Greber, B.J.; Grünberg, S.; Luo, J.; Fang, J.; Liu, Y.; Ranish, J.; Hahn, S.; Nogales, E. Structure of human TFIID and mechanism of TBP loading onto promoter DNA. Science 2018, 362, 1376. [CrossRef] [PubMed]

26. Liu, D.J.; Ishima, R.; Tong, K.I.; Bagby, S.; Kokubo, T.; Muhandiram, D.R.; Kay, L.E.; Nakatani, Y.; Ikura, M. Solution structure of a TBP-TAF(II)230 complex: Protein mimicry of the minor groove surface of the TATA box unwound by TBP. Cell 1998, 94, 573-583. [CrossRef]

27. Cheng, J.X.; Nevado, J.; Lu, Z.; Ptashne, M. The TBP-inhibitory domain of TAF145 limits the effects of nonclassical transcriptional activators. Curr. Biol. 2002, 12, 934-937. [CrossRef]

28. Boeger, H.; Griesenbeck, J.; Strattan, J.S.; Kornberg, R.D. Nucleosomes unfold completely at a transcriptionally active promoter. Mol. Cell 2003, 11, 1587-1598. [CrossRef]

29. Stark, H. GraFix: Stablization of fragile macromolecular complexes for single particle cryo-EM. Methods Enzymol. 2010, 481, 109-126. [CrossRef]

30. Kastner, B.; Fischer, N.; Golas, M.M.; Sander, B.; Dube, P.; Boehringer, D.; Hartmuth, K.; Deckert, J.; Hauer, F.; Wolf, E.; et al. GraFix: Sample preparation for single-particle electron cryomicroscopy. Nat. Methods 2008, 5, 53-55. [CrossRef]

31. Elmlund, D.; Elmlund, H. SIMPLE: Software for ab initio reconstruction of heterogeneous single-particles. J. Struct. Biol. 2012, 180, 420-427. [CrossRef] [PubMed]

32. Elmlund, D.; Davis, R.; Elmlund, H. Ab initio structure determination from electron microscopic images of single molecules coexisting in different functional states. Structure 2010, 18, 777-786. [CrossRef] [PubMed] 
33. Tang, G.; Peng, L.; Baldwin, P.R.; Mann, D.S.; Jiang, W.; Rees, I.; Ludtke, S.J. EMAN2: An extensible image processing suite for electron microscopy. J. Struct. Biol. 2007, 157, 38-46. [CrossRef] [PubMed]

34. Petterson, E.F.; Goddard, T.D.; Huang, C.C.; Couch, G.S.; Greenblatt, D.M.; Meng, E.C.; Ferrin, T.E. UCSF Chimera-A visualization system for exploratory research and analysis. J. Comput. Chem. 2004, 25, 1605-1612. [CrossRef] [PubMed]

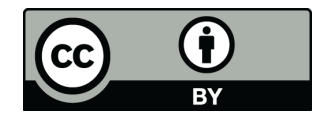

(C) 2019 by the authors. Licensee MDPI, Basel, Switzerland. This article is an open access article distributed under the terms and conditions of the Creative Commons Attribution (CC BY) license (http://creativecommons.org/licenses/by/4.0/). 